\title{
MODALITY OF REPRESENTATIONS
}

\author{
VLADIMIR L. POPOV* \\ To the memory of Bert Kostant
}

\begin{abstract}
We first establish several general properties of modality of algebraic group actions. In particular, we introduce the notion of a modality-regular action and prove that every visible action is modality-regular. Then, using these results, we classify irreducible linear representations of connected simple algebraic groups of every fixed modality $\leqslant 2$. Next, exploring a finer geometric structure of linear actions, we generalize to the case of any cyclically graded semisimple Lie algebra the notion of a packet (or a Jordan/decomposition class) and establish the properties of packets.
\end{abstract}

\section{Introduction}

The modality of a group action is the maximal number of parameters on which a family of orbits may depend. This notion, as a natural measure of complexity of a group action, goes back to V. I. Arnold's works on the theory of singularities in which the actions of diffeomorphism groups on the spaces of functions have been explored. V. I. Arnold and his collaborators succeeded in classifying the cases of a small modality ( 0 and 1$)$; this led to the famous lists of singularities that enjoy remarkable properties; see [A75].

The concept of modality naturally adapts to the setting of algebraic group actions on algebraic varieties [V86], [PV94, Sect. 5.2]. As in the theory of singularities, this concept allows one to systematically approach the classification problem of algebraic group actions by the degree of complexity. Here we explore the concept of modality in this setting.

In Section 2 we first discuss general properties of the modality of algebraic group actions; in particular, we introduce the notion of a modality-regular action and prove that every visible action is modality-regular. Then we consider the basic class of algebraic group actions, namely, that of the linear actions on (finite-dimensional) vector spaces. Guided by the analogy with V. I. Arnold's standpoint in the theory of singularities and with a view of obtaining the distinguished classes of linear actions, we consider the problem of classifying linear actions (representations) of a small modality and classify all irreducible representations of simple algebraic groups of every fixed modality $\leqslant 2$. They

* This work was supported by the Russian Foundation for Basic Research, project no. 1501-02158. 
are, indeed, turn out to be remarkable because of allowing some nice equivalent characterizations. Actually, for modality 0 the classification is not new: the definition of modality implies that the class of representations of modality 0 coincides with that of representations with finitely many orbits; it has been given much attention to the latter in the literature, in particular, all irreducible representations of reductive groups from this class have been listed [SK77], [K801], [KKY86]. For simple groups, however, the nice characterizations are possible, which for all reductive groups are no longer satisfied (see below Remark 2.17).

In Section 3, we explore the finer properties of the geometry of linear actions. Namely, a finer study of actions presupposes finding not only maximal number of parameters on which a family of orbits may depend (i.e., the modality), but also describing all maximal families of orbits (i.e., the sheets), and, where pos-

sible, presenting varieties as the disjoint unions of the finer families which have a standard structure and the better controlled geometric properties. For the adjoint representations of semisimple algebraic groups, and the isotropy representations of symmetric spaces, the solutions to these finer study problems are known, see [TY05, Chap. 39] and the references therein. In these cases, the latter finer families are the Jordan classes (also known as the decomposition classes and the packets). We generalize this notion (using the term "packet") to the case of any cyclically graded semisimple Lie algebra (or $\theta$-group, in the terminology of [V76], [K80 $]$ ). We describe all packets and explore their properties; in particular, we find their dimensions and modality.

As the base field we fix an algebraically closed field $k$ of characteristic zero. Below we freely use the standard notation and terminology of algebraic group theory and invariant theory from [B91] and [PV94], where also the proofs of unreferenced claims and/or the relevant references can be found. All considered actions of algebraic groups on algebraic varieties and all homomorphisms of algebraic groups (in particular, representations) are assumed to be algebraic (i.e., regular/morphic). Topological terms are related to the Zariski topology.

The results of Section 2 are partly announced in [P17 $]$.

\section{Modality}

2.1. Let $G$ be a connected algebraic group. We call any irreducible algebraic variety $F$ endowed with an action of $G$ such that all $G$-orbits in $F$ have the same dimension $d$ a family of $G$-orbits depending on

$$
\bmod (G: F):=\operatorname{dim} F-d
$$

parameters; the integer $\bmod (G: F)$ is called the modality of $F$. If $F \rightarrow F \vdots G$ is a rational quotient of this action (which exists by the Rosenlicht theorem), then

$$
\bmod (G: F)=\operatorname{dim} F \vdots G=\operatorname{tr} \operatorname{deg}_{k} k(F)^{G}
$$


and $F \vdots G$ may be informally viewed as the variety parametrizing typical $G$ orbits in $F$.

Given an algebraic variety $X$ endowed with an action of $G$, we denote by $\mathscr{F}(X)$ the set of all locally closed $G$-stable subsets of $X$ which are families. The integer

$$
\bmod (G: X):=\max _{F \in \mathscr{F}(X)} \bmod (G: F)
$$

is then called the modality of $X$. If $X$ is a vector space and the action is linear determined by a representation $\varrho: G \rightarrow \mathrm{GL}(X)$, then we call $\bmod (G: X)$ the modality of representation $\varrho$ and denote it by $\bmod \varrho$.

If $Y$ is an algebraic variety endowed with an action of a (not necessarily connected) algebraic group $H$ and if $H^{0}$ is the identity component of $H$, then, by definition ${ }^{1}$,

$$
\bmod (H: Y):=\bmod \left(H^{0}: Y\right) .
$$

Similarly, the modality of a representation of $H$ is defined as the modality of its restriction to $H^{0}$.

Recall that, for every integer $d$, the set $\{y \in Y \mid \operatorname{dim} H \cdot y \leqslant d\}$ is closed in $Y$. Whence, for every locally closed irreducible (not necessarily $H$-stable) subset $Z$ in $Y$, the subset

$$
Z^{\mathrm{reg}}:=\{z \in Z \mid \operatorname{dim} H \cdot z \geqslant \operatorname{dim} H \cdot y \text { for every } y \in Z\}
$$

is dense and open in $Z$.

The definition of modality implies that equality (3) still holds if $\mathscr{F}(X)$ is replaced by the set of all maximal (with respect to inclusion) families in $X$, i.e., by the sheets of $X$ [PV94, Sect. 6.10]. Recall that there are only finitely many sheets of $X$. If $X$ is irreducible, then $X^{\text {reg }}$ is a sheet, called regular, which is open and dense in $X$. It follows from (2) that

$$
\bmod \left(G: X^{\mathrm{reg}}\right)=\operatorname{tr} \operatorname{deg}_{k} k(X)^{G} .
$$

This implies that equality (3) still holds if $\mathscr{F}(X)$ is replaced by the set of all $G$ stable locally closed (or closed) subsets of $X$, and $\bmod (G: F)$ by $\operatorname{tr} \operatorname{deg}_{k} k(F)^{G}$.

The aforesaid shows that $\bmod (G: X)=0$ if and only if the set of all $G$-orbits in $X$ is finite.

If $G$ is reductive and $X$ is affine, then

$$
\begin{aligned}
\bmod (G: X) & \geqslant \bmod \left(G: X^{\mathrm{reg}}\right) \stackrel{(5)}{=} \operatorname{tr} \operatorname{deg}_{k} k(X)^{G} \\
& \geqslant \operatorname{tr} \operatorname{deg}_{k} k[X]^{G}=\operatorname{dim} X / / G .
\end{aligned}
$$

\footnotetext{
${ }^{1}$ This definition fixes the inaccuracy in [V86], [PV94, Sect. 5.2], where $\bmod (G: X)$ is defined by (3) for any $G$, not necessarily connected: as is easily seen, for a disconnected $G$, the set $\mathscr{F}(X)$ may be empty, so this definition should be corrected.
} 
2.2. The existence of regular sheets leads to defining the following naturally distinguished class of actions:

Definition 2.1. An action of a connected algebraic group $G$ on an irreducible algebraic variety $X$ is called modality-regular if $\bmod (G: X)=\bmod (G$ : $\left.X^{\text {reg }}\right)$. A linear representation $G \rightarrow \mathrm{GL}(V)$ is called modality-regular if it determines a modality-regular action of $G$ on $V$.

There are affine algebraic groups $G$ such that every action of $G$ is modalityregular. Here is their complete classification:

Theorem 2.2 (groups all actions of which are modality-regular). The following properties of a connected affine algebraic group $G$ are equivalent:

(i) all actions of $G$ on irreducible algebraic varieties are modality-regular;

(ii) for every irreducible algebraic variety $X$ and every action of $G$ on $X$ with a dense open $G$-orbit, there are only finitely many $G$-orbits in $X$;

(iii) $G$ is one of the following groups:

- a torus,

- a product of a torus and a group isomorphic to $\mathbf{G}_{a}$.

Proof. See $\left[\mathrm{P} 17_{2}\right]$.

2.3. However, a restriction of the class of actions under consideration may lead to an extension of the class of those groups for which all actions of this class are modality-regular. Apparently for the first time such a phenomenon was discovered in the following theorem:

Theorem 2.3. Let $G, B$, and $U$ be respectively a connected reductive algebraic group, a Borel subgroup of $G$, and a maximal unipotent subgroup of $G$. Then the restrictions to $B$ and $U$ of any action of $G$ on an irreducible algebraic variety are modality-regular.

Proof. See [V86, Thms. 2 and 3].

Remark 2.4. By Theorem 2.2, if $G$ is not abelian, then there are actions of $B$ which are not modality-regular. By Theorem 2.3, these $B$-actions can not be extended up to $G$-actions. If $\operatorname{rk} G \geqslant 2$, the same holds for $U$.

2.4. The next example of this phenomenon, in particular, shows that, apart from tori, there are other connected reductive algebraic groups for which every representation is modality-regular:

Theorem 2.5 (actions of $\mathrm{SL}_{2}$ ). Every action of $G=\mathrm{SL}_{2}$ on an irreducible quasiaffine algebraic variety $X$ is modality-regular.

Proof. Since $X$ is quasiaffine, there is an equivariant open embedding of $X$ in an affine algebraic variety endowed with an action of $G$; see [PV94, Thm. 1.6]. 
Therefore, we may (and shall) assume that $X$ is affine. Given a sheet $S \neq X^{\text {reg }}$, we need to show that

$$
\bmod (G: S) \leqslant \bmod \left(G: X^{\mathrm{reg}}\right)
$$

First we note that

$$
\operatorname{dim} X^{G} \leqslant \bmod \left(G: X^{\mathrm{reg}}\right) .
$$

Indeed, since $k[X]^{G}$ separates closed orbits, the restriction of the quotient morphism $X \rightarrow X / / G$ to $X^{G}$ is injective. Hence $\operatorname{dim} X^{G} \leqslant \operatorname{dim} X / / G$. This and (5) imply (8) because $\operatorname{dim} X / / G=\operatorname{tr} \operatorname{deg}_{k} k[X] \leqslant \operatorname{tr} \operatorname{deg}_{k} k(X)^{G}$.

In view of (8) we need to consider only the case where $S \cap X^{G}=\varnothing$. Assume that this equality holds. As is well-known, every one-dimensional homogeneous space of $G$ is projective (actually, isomorphic to $\mathbf{P}^{1}$ ). Whence, since every $G$ orbit in $X$ is quasiaffine, its dimension may be only $\operatorname{dim} G=3,2$, or 0 . This and $S \neq X^{\text {reg imply that }}$

$$
\operatorname{dim} G \cdot x=\left\{\begin{array}{ll}
2 & \text { if } x \in S, \\
3 & \text { if } x \in X^{\mathrm{reg}},
\end{array} \quad \text { and } \quad \operatorname{dim} S \leqslant \operatorname{dim} X-1 .\right.
$$

From (1), (9) we get $\bmod (G: S) \leqslant \operatorname{dim} X-3=\bmod \left(G: X^{\text {reg }}\right)$, whence (7).

Remark 2.6. By Theorem 2.2, the quasiaffinity condition in Theorem 2.5 can not be dropped.

2.5. Using Theorem 2.5, one computes the modality of every representation of $\mathrm{SL}_{2}$. Namely, let $\varrho_{n}$ be the $(n+1)$-dimensional linear representation of $\mathrm{SL}_{2}$ determining the natural $\mathrm{SL}_{2}$-module structure on the space of binary forms of degree $n$ over $k$. It is irreducible and every linear representation of $\mathrm{SL}_{2}$ is equivalent to a direct sum of such representations. Given a representation $\varrho$ and an integer $s>0$, we denote $s \varrho:=\varrho \oplus \cdots \oplus \varrho$ ( $s$ summands).

Corollary 2.7 (modality of $\mathrm{SL}_{2}$-representations). Let $\varrho: G \rightarrow \mathrm{GL}(V)$ be a linear representation of $G=\mathrm{SL}_{2}$. Then

$$
\bmod \varrho= \begin{cases}\operatorname{dim} \varrho-3 & \text { if } \varrho \neq s \varrho_{0} \oplus \varrho_{1}, s \varrho_{0} \oplus \varrho_{2}, s \varrho_{0}, \\ \operatorname{dim} \varrho-2 & \text { if } \varrho=s \varrho_{0} \oplus \varrho_{1}, s \varrho_{0} \oplus \varrho_{2}, \\ \operatorname{dim} \varrho & \text { if } \varrho=s \varrho_{0} .\end{cases}
$$

Proof. By Definition 2.1 and Theorem 2.5 we have $\bmod \varrho=\operatorname{dim} \varrho-\max _{v \in V} \operatorname{dim} G$. $v$. The integer $\max _{v \in V} \operatorname{dim} G \cdot v$ is computed [P74]. Whence the claim.

2.6. The following example shows that for every integer $n \geqslant 3$ there are linear representations of $G=\mathrm{SL}_{n}$ which are not modality-regular. In particular, $\mathrm{SL}_{2}$ in Theorem 2.5 cannot be replaced by $\mathrm{SL}_{n}$ for $n \geqslant 3$.

Example 2.8. Consider the natural action of $G$ on $k^{n}$ and the diagonal action of $G$ on $V:=k^{n} \oplus \cdots \oplus k^{n}$ ( $d$ summands). If $d \leqslant n-1$, there is an open $G$-orbit in $V$, so we have $\bmod \left(G: V^{\text {reg }}\right)=0$. On the other hand, 
for every nonzero $v, u \in k^{n}$ and $\lambda_{1}, \ldots, \lambda_{d-1}, \mu_{1}, \ldots, \mu_{d-1} \in k^{\times}$the elements $\left(v, \lambda_{1} v, \ldots, \lambda_{d-1} v\right) \in V$ and $\left(u, \mu_{1} u, \ldots, \mu_{d-1} u\right) \in V$ lie in the same $G$-orbit if and only if $\lambda_{i}=\mu_{i}$ for all $i$. This imples that $\bmod (G: V) \geqslant d-1$.

2.7. We shall now prove that all the representations from a certain important class are modality-regular; this will be then used in the proof of the classification results in Subsection 2.10.

Recall from [K75], $\left[\mathrm{K} 80_{1}\right]$ (see also $[\mathrm{PV} 94, \S 8]$ ) that a linear action of a reductive algebraic group $G$ on a vector space $V$ (and the corresponding representation $G \rightarrow \mathrm{GL}(V))$ is called visible if there are only finitely many $G$ orbits in the level variety of $k[V]^{G}$ in $V$ containing 0 . As a matter of fact, then automatically every level variety of $k[V]^{G}$ in $V$ contains only finitely many $G$-orbits [PV94, Cor. 3 of Prop. 5.1]. Extending this terminology to a more general setting, we introduce the following

Definition 2.9. An action of $G$ on an affine algebraic variety $X$ is called visible if every fiber of the categorical quotient

$$
\pi_{G, X}: X \rightarrow X / / G
$$

contains only finitely many $G$-orbits.

Theorem 2.10 (modality of visible actions). Every visible action of a reductive algebraic group $G$ on an irreducible affine algebraic variety $X$ enjoys the following properties:

(i) it is modality-regular;

(ii) $\bmod (G: X)=\operatorname{dim} X / / G$;

(iii) the induced action of $G$ on every closed $G$-stable subset of $X$ is visible.

Proof. First, we prove (iii). Let $Y$ be a $G$-stable closed subset of $X$. Then the set $Z:=\pi_{G, X}(Y)$ is closed in $X / / G$ and $\left.\pi_{G, X}\right|_{Y}: Y \rightarrow Z$ is the categorical quotient for the action of $G$ on $Y$; see [PV94, Sect. 4.4]. Every fiber of $\left.\pi_{G, X}\right|_{Y}$ is the intersection of $Y$ with a fiber of $\pi_{G, X}$; since the latter contains only finitely-many $G$-orbits, this intersection shares this property.

Now we prove (i) and (ii). Let $m_{G, X}:=\max _{x \in X} \operatorname{dim} G \cdot x$. For every $x \in$ $X^{\text {reg }}$ we then have $\operatorname{dim} G \cdot x=m_{G, X}$. Since the fiber $\pi_{G, X}^{-1}\left(\pi_{G, X}(x)\right)$ contains only finitely many orbits, the latter equality entails that the dimension of this fiber is equal to $m_{G, X}$. From this we infer that

$$
\bmod \left(G: X^{\mathrm{reg}}\right)=\operatorname{dim} X^{\mathrm{reg}}-m_{G, X}=\operatorname{dim} X-m_{G, X}=\operatorname{dim} X / / G
$$

(the second equality in (11) holds as $X^{\text {reg }}$ is open in $X$, and the third by the fiber dimension theorem).

Let $S$ be a sheet of $X$, let $\bar{S}$ be its closure in $X$, and let $Z:=\pi_{G, X}(\bar{S})=$ $\bar{S} / / G$. As $\bar{S}$ is a $G$-stable closed subset in $X$, the action of $G$ on $\bar{S}$ is visible by (iii). Therefore, replacing $X$ in (11) by $\bar{S}$ and taking into account that $S=\bar{S}^{\text {reg }}$, we obtain

$$
\bmod (G: S)=\operatorname{dim} Z
$$


Now the inclusion $Z \subseteq X / / G$ combined with (11) and (12), yields the inequality $\bmod \left(G: X^{\mathrm{reg}}\right) \geqslant \bmod (G: S)$. This completes the proof.

2.8. Theorem 2.10 is applicable to the class of so-called $\theta$-groups studied in [V76] (see also $\left[\mathrm{K} 80_{1}\right]$ ).

Namely, let $m$ be either a positive integer or $\infty$. Denote by $\mathbf{Z}_{m}$ the following additively written cyclic group of order $m$ : for $m<\infty$, the elements of $\mathbf{Z}_{m}$ are the integers between 0 and $m-1$, and the sum of $i$ and $j$ in $\mathbf{Z}_{m}$ is the remainder of dividing $i+j$ by $m$; by definition, $\mathbf{Z}_{\infty}=\mathbf{Z}$.

Consider a simply connected semisimple algebraic group $G$ and assume that its Lie algebra $\mathfrak{g}=\operatorname{Lie} G$ is $\mathbf{Z}_{m}$-graded:

$$
\mathfrak{g}=\bigoplus_{i \in \mathbf{Z}_{m}} \mathfrak{g}_{i}
$$

Then Aut $\mathfrak{g}$ contains a subgroup $\theta$, which, for $m<\infty$, is cyclic of order $m$, and, for $m=\infty$, a one-dimensional torus, such that (13) is the weight decomposition of $\mathfrak{g}$ with respect to the natural action of $\theta$.

The component $\mathfrak{g}_{0}$ in (13) is a reductive subalgebra of $\mathfrak{g}$. Let $G_{0}$ be a closed connected subgroup of $G$ with

$$
\text { Lie } G_{0}=\mathfrak{g}_{0} .
$$

Every $\mathfrak{g}_{i}$ in $(13)$ is $G_{0}$-stable with respect to the adjoint action, so one can consider $\mathfrak{g}_{i}$ as the $G_{0}$-module with respect to this action. As any $G_{0}$-module $\mathfrak{g}_{i}$ coincides with the $G_{0}$-module $\mathfrak{g}_{1}$ for another appropriate cyclic grading of $\mathfrak{g}$, when studying the orbital decompositions it suffices to explore only the $G_{0}$-module $\mathfrak{g}_{1}$. By [V76, Thm. 1], any two maximal linear subspaces of $\mathfrak{g}_{1}$ consisting of semisimple paiwise commuting elements are transformed to each other by $G_{0}$. These subspaces are called the Cartan subspaces and their mutual dimension is called the rank of the graded Lie algebra (13). The image of the adjoint representation $G_{0} \rightarrow \mathrm{GL}\left(\mathfrak{g}_{1}\right)$ is called the $\theta$-group associated with (13).

Theorem 2.11 (modality of $\theta$-group actions). In the above notation, the adjoint representation $\varrho: G_{0} \rightarrow \mathrm{GL}\left(\mathfrak{g}_{1}\right)$ is modality-regular and its modality is equal to the rank $r$ of the graded Lie algebra (13).

Proof. By [V76, Thm. 4], $\varrho$ is visible, and, by [V76, Thm. 5], $\operatorname{dim} \mathfrak{g}_{1} / / G_{0}=$ $r$. In view of this, the claim follows from Theorem 2.10.

Corollary 2.12 (case $m=1$ ). The adjoint representation of every connected semisimple algebraic group $G$ is modality-regular and its modality is equal to the rank of $G$.

Corollary 2.13 (case $m=2$ ). The isotropy representation of every symmetric space $X$ of a connected semisimple algebraic group is modality-regular and its modlity is equal to the rank of $X$. 
2.9. We now turn to classifying representations of a small modality. First, note that the following finiteness theorem can be considered as an argument in favor of considering the problem of classifying representations in terms of the magnitude of the modality.

Theorem 2.14 (finiteness for modality). For every connected simisimple algebraic group $G$ and every integer $m \geqslant 0$, there are only finitely many (up to equivalence) linear representations of $G$ of modality $m$.

Proof. First note that $G$ has only finitely many (up to equivalence) linear representations of any fixed dimension. Indeed, given the complete reducibility of representations, it suffices to prove this for irreducible representations. Denote by $\varrho(\lambda)$ the irreducible representation of $G$ with the highest weight $\lambda$ regarding a fixed Borel subgroup and its torus $T$. We may (and shall) assume that $G$ is simply connected; let then $\varpi_{1}, \ldots, \varpi_{r}$ be the fundamental weights of $T$. It follows from the Weyl formula for $\operatorname{dim} \varrho(\lambda)$, see [J62, Chap. VIII, $\S 4$, (41)], that $\operatorname{dim} \varrho\left(\sum_{i=1}^{r} n_{i} \varpi_{i}\right)<\operatorname{dim} \varrho\left(\sum_{i=1}^{r} m_{i} \varpi_{i}\right)$ if $0 \leqslant n_{i} \leqslant m_{i}$ for all $i$ and $n_{i_{0}}<m_{i_{0}}$ for some $i_{0}$. This implies the finiteness statement.

The claim of the theorem now follows from this statement combined with the inequality $\bmod \varrho \leqslant \operatorname{dim} \varrho$ that stems from the definition of $\bmod \varrho$.

2.10. In Theorem 2.15 below, we classify irreducible representations of connected simple algebraic groups of modalities 0,1 , and 2 . We use in this theorem the following agreements and notation.

Let $G$ be a a connected semisimple algebraic group and let $\pi: \widetilde{G} \rightarrow G$ be its universal covering. The map $\varrho \mapsto \widetilde{\varrho}:=\varrho \circ \pi$ is a bijection between the set of all representations of $G$ and the set of all representations of $\widetilde{G}$ factoring through $G$. This allows one to specify $\varrho$ by specifying $\widetilde{\varrho}$. If $\widetilde{\varrho}$ is irreducible, it is uniquely up to equivalence determined by its highest weight $\lambda$ (with respect to a fixed Borel subgroup $B$ of $\widetilde{G}$ and its maximal torus $T$ ). Given this, we denote $\varrho$ (considered up to equivalence) by $(\mathrm{R}, \lambda)$, where $\mathrm{R}$ is the type of the root system of $\widetilde{G}$. The fundamental weights of $\widetilde{G}$ with respect to the pair $(B, T)$ are denoted by $\varpi_{1}, \ldots, \varpi_{r}$; we use their Bourbaki numbering [B68]. For $\mathrm{R}=\mathrm{A}_{r}, \mathrm{~B}_{r}, \mathrm{C}_{r}, \mathrm{D}_{r}$, we assume that, respectively, $r \geqslant 1,3,2,4$. The group of characters of $T$ is considered in additive notation. The representation contragredient to $\varrho$ is denoted by $\varrho^{*}$.

Theorem 2.15 (irreducible representations of simple algebraic groups of modalities 0,1,2). Let $G$ be a connected simple algebraic group and let $V$ be a $G$-module determined by a nontrivial irreducible representation $\varrho: G \rightarrow \mathrm{GL}(V)$.

$\left(\mathrm{M}_{1}\right)$ The condition $\bmod \varrho=0$ is equivalent to either of the following:

(i) $k[V]^{G}=k$;

(ii) the action of $G$ on $V$ is nonstable;

(iii) $\varrho$ or $\varrho^{*}$ is contained in the following list:

$$
\left(\mathrm{A}_{r}, \varpi_{1}\right) ;\left(\mathrm{A}_{r}, \varpi_{2}\right), r \geqslant 4 \text { is even; }\left(\mathrm{C}_{r}, \varpi_{1}\right) ;\left(\mathrm{D}_{5}, \varpi_{5}\right) .
$$


$\left(\mathrm{M}_{2}\right)$ The condition $\bmod \varrho=1$ is equivalent to either of the following:

(i) $\operatorname{tr} \operatorname{deg}_{k} k[V]^{G}=1$;

(ii) $V / / G=\mathbf{A}^{1}$;

(iii) $\varrho$ or $\varrho^{*}$ is contained in the following list:

$$
\begin{gathered}
\left(\mathrm{A}_{r}, 2 \varpi_{1}\right) ;\left(\mathrm{A}_{r}, \varpi_{2}\right), r \geqslant 3 \text { is odd } ;\left(\mathrm{B}_{r}, \varpi_{1}\right) ;\left(\mathrm{D}_{r}, \varpi_{1}\right) ; \\
\left(\mathrm{A}_{1}, 3 \varpi_{1}\right) ;\left(\mathrm{A}_{5}, \varpi_{3}\right) ;\left(\mathrm{A}_{6}, \varpi_{3}\right) ;\left(\mathrm{A}_{7}, \varpi_{3}\right) ;\left(\mathrm{B}_{3}, \varpi_{3}\right) ;\left(\mathrm{B}_{4}, \varpi_{4}\right) ;\left(\mathrm{B}_{5}, \varpi_{5}\right) ; \\
\left(\mathrm{C}_{2}, \varpi_{2}\right) ;\left(\mathrm{C}_{3}, \varpi_{3}\right) ;\left(\mathrm{D}_{6}, \varpi_{6}\right) ;\left(\mathrm{D}_{7}, \varpi_{7}\right) ;\left(\mathrm{G}_{2}, \varpi_{1}\right) ;\left(\mathrm{E}_{6}, \varpi_{1}\right) ;\left(\mathrm{E}_{7}, \varpi_{7}\right) .
\end{gathered}
$$

$\left(\mathrm{M}_{3}\right)$ The condition $\bmod \varrho=2$ is equivalent to either of the following:

(i) $\operatorname{tr} \operatorname{deg}_{k} k[V]^{G}=2$;

(ii) $V / / G=\mathbf{A}^{2}$;

(iii) $\varrho$ or $\varrho^{*}$ is contained in the following list:

$$
\begin{gathered}
\left(\mathrm{A}_{1}, 4 \varpi_{1}\right) ;\left(\mathrm{A}_{2}, \varpi_{1}+\varpi_{2}\right) ;\left(\mathrm{A}_{2}, 3 \varpi_{1}\right) ;\left(\mathrm{B}_{6}, \varpi_{6}\right) ; \\
\left(\mathrm{C}_{2}, 2 \varpi_{1}\right) ;\left(\mathrm{C}_{3}, \varpi_{2}\right) ;\left(\mathrm{F}_{4}, \varpi_{4}\right) ;\left(\mathrm{G}_{2}, \varpi_{2}\right) .
\end{gathered}
$$

If $\bmod \varrho \leqslant 2$, then $\varrho$ is modality-regular.

First we prove the following

Lemma 2.16. Let $G$ be a connected semisimple algebraic group and let $V$ be a $G$-module such that $\bmod (G: V) \leqslant 2$. Then $k[V]^{G}$ is a free $k$-algebra.

Proof. By (6), we have $\operatorname{dim} V / / G \leqslant 2$. If $\operatorname{dim} V / / G=0$, then $k[V]^{G}=k$. If $\operatorname{dim} V / / G=1$, then it follows from the Lüroth theorem that $V / / G=\mathbf{A}^{1}$; see [P80, Prop. 12] (this is true for any reductive $G$ ). If $\operatorname{dim} V / / G=2$, then,

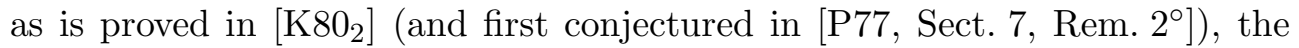
assumption that $G$ is connected semisimple entails $V / / G=\mathbf{A}^{2}$ (cf. also [PV94, Sect. 8.4]). This completes the proof.

Proof of Theorem 2.15. By Lemma 2.16, if $\bmod \varrho \leqslant 2$, then $\varrho$ is cofree (i.e., $k[V]^{G}$ is a free $k$-algebra). The list of all cofree irreducible representations of connected simple algebraic group is obtained in [KPV76] (see also Summary Table in [PV94, pp. 259-262]). This leads to determining which representations from this list have modality $\leqslant 2$. Comparing this list with the list of all irreducible visible representations obtained in $\left[\mathrm{K} 80_{1}\right.$, Thm. 1] shows that every representation $\varrho$ from the former list is visible. Whence, by Theorem 2.10 , it is modality-regular and $\bmod \varrho=\operatorname{dim} V / / G$. Since the integers $\operatorname{dim} V / / G$ are known (they are specified in the fifth column of the Summary Table in [PV94, pp. 259-262]), this yields the lists in $\left(\mathrm{M}_{1}\right)(\mathrm{iii}),\left(\mathrm{M}_{2}\right)(\mathrm{iii})$, and $\left(\mathrm{M}_{3}\right)(\mathrm{iii})$, thereby proving all the claims except $\left(\mathrm{M}_{1}\right)$ (ii). By [P71, Thm. 1], $\varrho$ is nonstable if and only if the $G$-stabilizer of a point in general position in $V$ is nonreductive. Since the stabilizers of points in general position for the representations from this list are known as well (they are specified in the fourth column of the Summary Table in [PV94, pp. 259-262]), applying this criterion yields that $\left(\mathrm{M}_{1}\right)$ (ii) and $\left(\mathrm{M}_{1}\right)$ (iii) are equivalent. This completes the proof. 


\section{Remarks 2.17.}

1. In the following statements, the assumptions of the irreducibility of $\rho$ and the simplicity of $G$ are essential:

(a) In $\left(\mathrm{M}_{1}\right)$, in the claims that $k[V]=k$ or the nonstability of the action of $G$ on $V$ implies $\bmod \varrho=0$. Examples:

- If $\rho=2\left(\mathrm{~A}_{r}, \varpi_{1}\right)$ and $r \geqslant 2$, then $k[V]^{G}=k$, the action of $G$ on $V$ is nonstable, and $\bmod \rho>0$; see Example 2.8.

- If $\varrho=\left(\mathrm{A}_{r}, \varpi_{2}\right) \otimes\left(\mathrm{A}_{1}, \varpi_{1}\right)$, where $r \geqslant 8$ is even, then $k[V]^{G}=k$, see [L89], and the action of $G$ on $V$ in nonstable, see [PV94, Thm. 3.3, Cor. of Thm. 2.3], but the number of $G$-orbits in $V$ is infinite (equivalently, $\bmod \varrho>0$ ); see

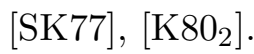

(b) In $\left(\mathrm{M}_{2}\right)$, in the claim that $\operatorname{tr} \operatorname{deg}_{k} k[V]^{G}=1$ is equivalent to $\bmod \varrho=$ 1. For example, if $\rho=(r+1)\left(\mathrm{A}_{r}, \varpi_{1}\right)$ and $r \geqslant 2$, then $\operatorname{tr} \operatorname{deg}_{k} k[V]^{G}=1$, and $\bmod \rho \geqslant r$; see Example 2.8.

(c) In $\left(\mathrm{M}_{3}\right)$, in the claim that $\bmod \varrho=2$ implies $\operatorname{tr} \operatorname{deg}_{k} k[V]^{G}=2$. For example, if $\rho=3\left(\mathrm{~A}_{r}, \varpi_{1}\right)$ and $r \geqslant 3$, then it is not difficult to see that $k[V]^{G}=k$ and $\bmod \rho=2$.

The representations $\rho$ specified in (a), (b), (c) are not modality-regular.

2. Arguing along the same lines, one can extend the classifications obtained in Theorem 2.15 up to the classifications of all irreducible castling reduced representations of connected semisimple algebraic groups of every fixed modality $\leqslant 2$. The reason being that the complete list of cofree irreducible castling reduced representations of connected semisimple algebraic groups is known [L89]. Comparing it with that of visible representations obtained in [SK77], $\left[\mathrm{K} 80_{1}\right]$, [KKY86], one ascertains that the majority of representations in this list (all but two) are visible, therefore, for them, the modality is given by Theorem 2.10 (the integers $\operatorname{dim} V / / G$ are specified in the fifth column of [L89, Tabelle]). To the remaining two representations one applies the ad hoc considerations.

3. The arguments from the proof of Theorem 2.15 yield the modalities of all cofree irreducible representations of connected simple algebraic groups.

2.11. We conclude this section with a statement, which in some cases helps to practically determine the modality.

Lemma 2.18. Let $X$ be an algebraic variety endowed with an action of an algebraic group $G$. Let $\left\{C_{i}\right\}_{i \in I}$ be a collection of subsets of $X$ such that

(i) I is finite;

(ii) $\bigcup_{i \in I} C_{i}=X$;

(iii) the closure $\overline{C_{i}}$ of $C_{i}$ in $X$ is irreducible for every $i \in I$;

(iv) every $C_{i}$ is $G$-stable;

(v) all $G$-orbits in $C_{i}$ have the same dimension $d_{i}$ for every $i \in I$.

Then the following hold: 
(a) $\bmod (G: X)=\max _{i \in I}\left(\operatorname{dim} \overline{C_{i}}-d_{i}\right)$;

(b) if $X$ is irreducible, then $X=\overline{C_{i_{0}}}$ for some $i_{0}$, and $\bmod \left(G: X^{\mathrm{reg}}\right)=$ $\operatorname{dim} X-d_{i_{0}}$.

Proof. By (iii), we have a family ${\overline{C_{i}}}^{\text {reg }}$, and (v) implies $C_{i} \subseteq{\overline{C_{i}}}^{\text {reg }}$. Whence

$$
\bmod \left(G:{\overline{C_{i}}}^{\text {reg }}\right)=\operatorname{dim} \overline{C_{i}}-d_{i} .
$$

From $(3)$ and $(15)$, we infer that $\bmod (G: X) \geqslant \max _{i \in I}\left(\operatorname{dim} \bar{C}_{i}-d_{i}\right)$. To prove the opposite inequality let $Z \in \mathscr{F}(X)$ be a family of $s$-dimensional $G$-orbits such that $\bmod (G: X)=\operatorname{dim} Z-s$ and let $J:=\left\{i \in I \mid Z \cap C_{i} \neq \varnothing\right\}$. By (ii), we have $Z=\bigcup_{j \in J}\left(Z \cap \overline{C_{j}}\right)$. Since $Z$ is irreducible and, by (i), $J$ is finite, there is $j_{0} \in J$ such that $Z \subseteq \overline{C_{j_{0}}}$. As $Z \cap C_{j_{0}} \neq \varnothing$, we have $s=d_{j_{0}}$. Therefore, $\bmod (G: X)=\operatorname{dim} Z-s \leqslant \operatorname{dim} \overline{C_{j_{0}}}-d_{j_{0}}$. This proves (a).

By (ii), $\bigcup_{i \in I} \overline{C_{i}}=X$. If $X$ is irreducible, then, in view of (i), this equality implies the existence of $i_{0}$ such that $X=\overline{C_{i_{0}}}$. This and (15) prove (b).

Example 2.19. Consider the graded semisimple Lie algebra (13) and the adjoint representation $\varrho: G_{0} \rightarrow \mathrm{GL}\left(\mathfrak{g}_{1}\right)$. In Section 3 we define a collection $\left\{C_{i}\right\}_{i \in I}$ of subsets of $\mathfrak{g}_{1}$ called packets (see below Definition 3.6). In Propositions 3.8, 3.9, 3.13 below we prove that this collection satisfies all conditions (i) $-(\mathrm{v})$ from Lemma 2.18, and in Corollary 3.14 below we compute $\bmod \left(G_{0}: \overline{C_{i}}\right)$ for every $i$. The integer $\max _{i \in I} \bmod \left(G_{0}: \overline{C_{i}}\right)$ turns then out to be equal to the rank of the graded Lie algebra (13). By Lemma 2.18, this agrees with Theorem 2.11.

Corollary 2.20. Let $Y$ and $F$ be the algebraic varieties endowed with the actions of an algebraic group $G$. Consider the diagonal action of $G$ on $X:=$ $Y \times F$. If $G$ acts on $F$ trivially, then

(i) $\bmod (G: X)=\bmod (G: Y)+\operatorname{dim} F$;

(ii) the action of $G$ on $Y$ is modality-regular if and only if the action of $G$ on $X$ is modality-regular.

Proof. Let $\left\{S_{i}\right\}_{i \in I}$ be the collection of all sheets of $Y$. The claim then follows from Lemma 2.18 applied to $\left\{C_{i}\right\}_{i \in I}$ where $C_{i}:=S_{i} \times F$.

\section{Packets in cyclically graded semisimple Lie algebras}

In this section we explore the finer geometric properties of $\theta$-group actions.

3.1. Besides the notation of Subsection 2.8 below we also use the following:

- If $\mathfrak{a}$ and $\mathfrak{b}$ are nonempty subsets of $\mathfrak{g}$, then $\mathfrak{a}^{\mathfrak{b}}$ is the centralizer of $\mathfrak{b}$ in $\mathfrak{a}$,

$$
\mathfrak{a}^{\mathfrak{b}}:=\{x \in \mathfrak{a} \mid[x, y]=0 \text { for all } y \in \mathfrak{b}\},
$$

and $[\mathfrak{a}, \mathfrak{b}]$ is the $k$-linear span of all $[x, y]$, where $x \in \mathfrak{a}, y \in \mathfrak{b}$.

- The center of $\mathfrak{a}$, i.e., $\mathfrak{a}^{\mathfrak{a}}$, is denoted by $\mathfrak{z}(\mathfrak{a})$.

- For any subset $\mathfrak{s}$ in $\mathfrak{g}$, we put $\mathfrak{s}_{i}:=\mathfrak{s} \cap \mathfrak{g}_{i}$ (the case $\mathfrak{s} \cap \mathfrak{g}_{i}=\varnothing$ is not excluded). 
The following facts, used below, are proved in [V76]:

There exists a nondegenerate $G$-invariant and $\theta$-invariant scalar multiplication $\mathfrak{g} \times \mathfrak{g} \rightarrow k,(x, y) \mapsto\langle x, y\rangle$.

If $x=x_{\mathrm{s}}+x_{\mathrm{n}}$ is the Jordan decomposition of an element $x \in \mathfrak{g}$ with $x_{\mathrm{s}}$ semisimple and $x_{\mathrm{n}}$ nilpotent, then $x \in \mathfrak{g}_{i}$ entails $x_{\mathrm{s}}, x_{\mathrm{n}} \in \mathfrak{g}_{i}$.

There are only finitely many nilpotent $G_{0}$-orbits in every $\mathfrak{g}_{i}$.

3.2. We first consider the following general construction and introduce the related terminology and notation.

Definition 3.1. Let $M$ be a nonempty set and let $F$ be a nonempty set of functions $M \rightarrow k$. Define the equivalence relation $\sim_{F}$ on $M$ by

$$
x \sim_{F} y \Longleftrightarrow \text { for every } \alpha \in F \text {, either } \alpha(x)=\alpha(y)=0 \text { or } \alpha(x) \alpha(y) \neq 0 .
$$

The equivalence classes of $\sim_{F}$ are called the cells of $\sim_{F}$. The set of all cells of $\sim_{F}$ is denoted by $\mathscr{C}_{F}(M)$.

Proposition 3.2. Let $V$ be a finite-dimensional vector space over $k$ and let $F$ be a finite subset of the dual space $V^{*}$.

(i) For every linear subspace $L$ in $V^{*}$, the set

$$
C_{L}:=\left\{\begin{array}{l|l}
v \in V & \begin{array}{l}
\alpha(v)=0 \text { for all } \alpha \in F \cap L, \\
\beta(v) \neq 0 \text { for all } \beta \in F \backslash L .
\end{array}
\end{array}\right\}
$$

is nonempty.

(ii) A subset of $V$ is a cell of $\sim_{F}$ if and only if it is $C_{L}$ for some $L$.

(iii) The closure $\overline{C_{L}}$ of $C_{L}$ in $V$ is the linear subspace

$$
\{v \in V \mid \alpha(v)=0 \text { for all } \alpha \in F \cap L\},
$$

and the complement of $C_{L}$ in $\overline{C_{L}}$ is the union of hyperplanes

$$
\bigcup_{\beta \in F \backslash L}\left\{v \in \overline{C_{L}} \mid \beta(v)=0\right\} .
$$

(iv) The set $\mathscr{C}_{F}(V)$ is finite.

Proof. (i) In view of (16), without changing $C_{L}$, we may (and shall) assume that $L$ is the linear span of $L \cap F$. If $L=V^{*}$, then $C_{L}=0$. Let $L \neq V^{*}$; then the dimension of the linear subspace (17) is positive. The restriction of every $\beta \in F \backslash L$ to it is nonzero. For, otherwise, $\operatorname{rk}((F \cap L) \cup \beta)=\operatorname{rk}(F \cap L)$, whence $\beta$ lies in the linear span of $F \cap L$, i.e., in $L,-$ a contradiction. Thus the locus of zeros of the restriction of $\beta$ to the linear subspace (17) is its proper linear subspace. Whence $C_{L}$ is nonempty.

(ii) It follows directly from the definitions of $\sim_{F}$ and $C_{L}$ that $C_{L}$ is a cell of $\sim_{F}$. Conversely, let $C$ be a cell of $\sim_{F}$ in $V$. If $L$ is the linear span of $\left\{\alpha \in F|\alpha|_{C}=0\right\}$, then Definition 3.1 implies that every $\beta \in F \backslash L$ vanishes nowhere on $C$. Hence $C \subseteq C_{L}$. Since, by (i), $C_{L}$ is a cell, this yields $C=C_{L}$.

(iii) This follows from (i).

(iv) In view of (i), this follows from the finiteness of $F$. 
Corollary 3.3. Every cell of $\sim_{F}$ in $V$ is an irreducible smooth rational affine algebraic variety locally closed in $V$.

3.3. We now fix a maximal torus $\mathfrak{t}$ of $\mathfrak{g}$ such that $\mathfrak{t}_{1}$ is a Cartan subspace of $\mathfrak{g}_{1}$ (since the minimal algebraic subalgebra of a Cartan subspace is a torus, such a $\mathfrak{t}$ exists). Let $R \subset \mathfrak{t}^{*}$ be the root system of $\mathfrak{g}$ with respect to $\mathfrak{t}$. As usual, if $\alpha \in R$, then $\mathfrak{g}_{\alpha}:=\{x \in \mathfrak{g} \mid[t, x]=\alpha(t) x$ for every $t \in \mathfrak{t}\}$.

We consider the cells of $\sim_{R}$ in $\mathfrak{t}$.

Proposition 3.4. Let $\mathfrak{c}$ be a cell of $\sim_{R}$ in $\mathfrak{t}$ and let

$$
R_{\mathfrak{c}}:=\left\{\alpha \in R|\alpha|_{\mathfrak{c}}=0\right\} .
$$

(i) For every nonempty subset $\mathfrak{s} \subseteq \mathfrak{c}$, the following hold:

$\left(\mathrm{i}_{1}\right) \mathfrak{g}^{\mathfrak{s}}$ is a reductive subalgebra of $\mathfrak{g}$ with the maximal torus $\mathfrak{t}$ and the $\mathfrak{t}$-root decomposition

$$
\mathfrak{g}^{\mathfrak{s}}=\mathfrak{t} \oplus\left(\bigoplus_{\alpha \in R_{\mathfrak{c}}} \mathfrak{g}_{\alpha}\right) .
$$

$\left(\mathrm{i}_{2}\right)$ the center $\mathfrak{z}\left(\mathfrak{g}^{\mathfrak{s}}\right)$ of $\mathfrak{g}^{\mathfrak{s}}$ is $\overline{\mathfrak{c}}$.

$\left(\mathrm{i}_{3}\right)$ the commutator ideal $\left[\mathfrak{g}^{\mathfrak{s}}, \mathfrak{g}^{\mathfrak{s}}\right]$ of $\mathfrak{g}^{\mathfrak{s}}$ is $\overline{\mathfrak{c}}^{\perp} \oplus\left(\bigoplus_{\alpha \in R_{\mathfrak{c}}} \mathfrak{g}_{\alpha}\right)$, where $\overline{\mathfrak{c}}^{\perp}$ is the orthogonal complement of $\overline{\mathfrak{c}}$ in $\mathfrak{t}$ with respect to $\left.\langle\cdot, \cdot\rangle\right|_{\mathfrak{t}}$.

(ii) If $t \in \mathfrak{g}$ is a semisimple element such that $\mathfrak{g}^{t}=\mathfrak{g}^{\mathfrak{c}}$, then $t \in \mathfrak{c}$.

Proof. ( $\left.\mathrm{i}_{1}\right)$ Since the elements of $\mathfrak{s}$ are semisimple and pairwise commute, the Lie algebra $\mathfrak{g}^{\mathfrak{s}}$ is reductive. As $\mathfrak{g}^{\mathfrak{s}}=\bigcap_{c \in \mathfrak{s}} \mathfrak{g}^{c}$, to prove (18) it suffices to show that (18) holds for $\mathfrak{s}$ being a single element $c \in \mathfrak{c}$. Let $x \in \mathfrak{g}$. The root decomposition of $\mathfrak{g}$ with respect to $\mathfrak{t}$,

$$
\mathfrak{g}=\mathfrak{t} \oplus\left(\bigoplus_{\alpha \in R} \mathfrak{g}_{\alpha}\right),
$$

yields $x=t+\sum_{\alpha \in R} x_{\alpha}$ for some $t \in \mathfrak{t}, x_{\alpha} \in \mathfrak{g}_{\alpha}$. Hence $[c, x]=\sum_{\alpha \in R} \alpha(c) x_{\alpha}$. Therefore, $x \in \mathfrak{g}^{c}$ if and only if $\alpha(c)=0$ for every $\alpha$ such that $x_{\alpha} \neq 0$. The condition $c \in \mathfrak{c}$ implies that $\alpha(c)=0$ if and only if $\alpha \in R_{\mathfrak{c}}$. Whence $x \in \mathfrak{g}^{c}$ if and only if $x_{\alpha}=0$ for all $\alpha \notin R_{\mathfrak{c}}$. This and (19) prove (i).

$\left(\mathrm{i}_{2}\right)$ Since the center of any reductive Lie algebra lies in every maximal torus of the latter, $\left(\mathrm{i}_{1}\right)$ and Proposition 3.2 entail

$$
\begin{aligned}
\mathfrak{z}\left(\mathfrak{g}^{\mathfrak{s}}\right) & =\left\{t \in \mathfrak{t} \mid\left[t, \mathfrak{g}_{\alpha}\right]=\alpha(t) \mathfrak{g}_{\alpha}=0 \text { for each } \alpha \in R_{\mathfrak{c}}\right\} \\
& =\left\{t \in \mathfrak{t} \mid \alpha(t)=0 \text { for each } \alpha \in R_{\mathfrak{c}}\right\}=\overline{\mathfrak{c}} .
\end{aligned}
$$

$\left(\mathrm{i}_{3}\right)$ This follows from $(18)$ and $\left(\mathrm{i}_{2}\right)$.

(ii) As $\mathfrak{t}$ lies in $\mathfrak{g}^{\mathfrak{c}}$ by $\left(\mathfrak{i}_{1}\right)$, the equality $\mathfrak{g}^{t}=\mathfrak{g}^{\mathfrak{c}}$ entails that $t$ commutes with $\mathfrak{t}$. As $t$ is semisimple and $\mathfrak{t}$ is a maximal torus, this shows that $t \in \mathfrak{t}$. Let $\mathfrak{c}^{\prime}$ be the unique cell in $\mathfrak{t}$ containing $t$. From $\left(i_{2}\right)$ we obtain $\overline{\mathfrak{c}^{\prime}}=\mathfrak{z}\left(\mathfrak{g}^{t}\right)=\mathfrak{z}\left(\mathfrak{g}^{\mathfrak{c}}\right)=\overline{\mathfrak{c}}$. By Proposition 3.2(ii),(iii), this yileds $\mathfrak{c}^{\prime}=\mathfrak{c}$.

Corollary 3.5. Every two nonempty subsets of any cell of $\sim_{R}$ in $\mathfrak{t}$ have the same centralizers in $\mathfrak{g}$. 
3.4. The decomposition $\mathfrak{t}=\bigsqcup_{\mathfrak{c} \in \mathscr{C}_{R}(\mathfrak{t})} \mathfrak{c}$ yields the decomposition

$$
\mathfrak{t}_{1}=\bigsqcup_{\mathfrak{c} \in \mathscr{C}_{R}(\mathfrak{t})} \mathfrak{c}_{1}
$$

Definition 3.6. For every cell $\mathfrak{c} \in \mathscr{C}_{R}(\mathfrak{t})$ such that $\mathfrak{c}_{1} \neq \varnothing$ and every nilpotent element $n \in \mathfrak{g}_{1}^{\mathfrak{c}}$, the set $G_{0} \cdot\left(\mathfrak{c}_{1}+n\right)$ is called a packet in $\mathfrak{g}_{1}$.

Remark 3.7. By [TY05, Prop. 39.1.5] (cf. also Proposition 3.10 below), for $m=1$ in (13), Definition 3.6 is equivalent to the usual definition of a packet (also known as Jordan/decomposition class) in $\mathfrak{g}$; see [TY05, 39.1.3] and the references in [P08].

3.5. In this subsection we prove some basic properties of packets in $\mathfrak{g}$. Below, for every locally closed subset $X$ in $\mathfrak{g}_{1}$, the notation $X^{\text {reg }}$ refers to the action of $G_{0}$ on $\mathfrak{g}_{1}$ if otherwise is not stated.

Proposition 3.8. The union of all packets in $\mathfrak{g}_{1}$ coincides with $\mathfrak{g}_{1}$.

Proof. Let $x \in \mathfrak{g}_{1}$; we should show that $x$ lies in a packet. We have $x_{\mathrm{s}}, x_{\mathrm{n}} \in$ $\mathfrak{g}_{1}$. Definition 3.6 shows that packets in $\mathfrak{g}_{1}$ are $G_{0}$-stable. On the other hand, since $\mathfrak{t}_{1}$ is a Cartan subspace in $\mathfrak{g}_{1}$ (by the choice of $\mathfrak{t}$ ), the $G_{0}$-orbit of $x_{\mathrm{s}}$ intersects $\mathfrak{t}_{1}$, see [V76, Cor. of Thm. 1]. Hence when proving that $x$ lies in a packet, we can (and shall) assume that $x_{\mathrm{S}} \in \mathfrak{t}_{1}$. In view of (20), there exists a cell $\mathfrak{c} \in \mathscr{C}_{R}(\mathfrak{t})$ such that $x_{\mathrm{s}} \in \mathfrak{c}_{1}$. By the definition of the Jordan decomposition of $x$, we have $x_{\mathrm{n}} \in \mathfrak{g}_{1}^{x_{\mathrm{s}}}$. In view of Corollary 3.5 , we have $\mathfrak{g}_{1}^{x_{\mathrm{s}}}=\mathfrak{g}_{1}^{\mathfrak{c}}$. Hence, by Definition $3.6, G_{0} \cdot\left(\mathfrak{c}_{1}+x_{\mathrm{n}}\right)$ is a packet in $\mathfrak{g}_{1}$; clearly $x$ lies in it.

Proposition 3.9. There are only finitely many packets in $\mathfrak{g}_{1}$.

Proof. Since the set $\mathscr{C}_{R}(\mathfrak{t})$ is a finite by Proposition 3.2(iv), it suffices to show that, for any given cell $\mathfrak{c} \in \mathscr{C}_{R}(\mathfrak{t})$ with $\mathfrak{c}_{1} \neq \varnothing$, there are only finitely many packets of the form $G_{0} \cdot\left(\mathfrak{c}_{1}+n\right)$ where $n$ is a nilpotent element of $\mathfrak{g}_{1}^{\mathfrak{c}}$.

To this end, consider the Lie subalgebra $\mathfrak{g}^{\mathfrak{c}_{1}}$ of $\mathfrak{g}$. By Proposition 3.4(i), it is reductive. As $\mathfrak{g}_{1}$ is a weight space of $\theta$, the inclusion $\mathfrak{c}_{1} \subseteq \mathfrak{g}_{1}$ entails that $\mathfrak{g}^{\mathfrak{c}_{1}}$ is $\theta$-stable, i.e., $\mathfrak{g}^{\mathfrak{c}_{1}}$ is a graded subalgebra of the graded Lie algebra $\mathfrak{g}$. In views of Corollary 3.5, we have $\mathfrak{g}^{\mathfrak{c}_{1}}=\mathfrak{g}^{\mathfrak{c}}$.

Let $S$ be a closed connected subgroup of $G_{0}$ such that

$$
\text { Lie } S=\mathfrak{g}_{0}^{\mathfrak{c}} .
$$

By [V76, Prop. 2], there are only finitely many orbits of the adjoint action of $S$ on the variety of nilpotent elements of $\mathfrak{g}_{1}^{\mathfrak{c}}$. Let $n_{1}, \ldots, n_{d}$ be the representatives of these orbits.

Now consider a packet $G_{0} \cdot\left(\mathfrak{c}_{1}+n\right)$, where $n$ is a nilpotent element of $\mathfrak{g}_{1}^{\mathfrak{c}}$. By the aforesaid, $S \cdot n=S \cdot n_{i}$ for some $i$. Since, in view of (21), every element of $\mathfrak{c}_{1}$ is a fixed point of $S$, this implies that

$$
S \cdot\left(\mathfrak{c}_{1}+n\right)=S \cdot\left(\mathfrak{c}_{1}+n_{i}\right) .
$$


In turn, since $S$ is a subgroup of $G_{0}$, from Definition 3.6 and (22) we obtain

$$
G_{0} \cdot\left(\mathfrak{c}_{1}+n\right)=G_{0} S \cdot\left(\mathfrak{c}_{1}+n\right) \stackrel{(22)}{=} G_{0} S \cdot\left(\mathfrak{c}_{1}+n_{i}\right)=G_{0} \cdot\left(\mathfrak{c}_{1}+n_{i}\right) .
$$

This proves the required finiteness statement.

Proposition 3.10. For any $x, y \in \mathfrak{g}_{1}$ the following properties are equivalent:

(P) There exists a packet $G_{0} \cdot\left(\mathfrak{c}_{1}+n\right)$ in $\mathfrak{g}_{1}$ containing both $x$ and $y$.

$(\mathrm{J})$ There exists an element $g \in G_{0}$ such that

$$
g \cdot \mathfrak{g}^{x_{\mathrm{s}}}=\mathfrak{g}^{g \cdot x_{\mathrm{s}}}=\mathfrak{g}^{y_{\mathrm{s}}} \quad \text { and } \quad g \cdot x_{\mathrm{n}}=y_{\mathrm{n}} .
$$

Proof. $(\mathrm{P}) \Rightarrow(\mathrm{J})$ Let $x, y \in G_{0} \cdot\left(\mathfrak{c}_{1}+n\right)$. By Definition 3.6, there are $c_{1}, c_{2} \in \mathfrak{c}_{1}$ and $g_{1}, g_{2} \in G_{0}$ such that $g_{1} \cdot x=c_{1}+n, g_{2} \cdot y=c_{2}+n$. Hence

$$
\begin{aligned}
& g_{1} \cdot x_{\mathrm{s}}=\left(g_{1} \cdot x\right)_{\mathrm{s}}=c_{1}, \quad g_{1} \cdot x_{\mathrm{n}}=\left(g_{1} \cdot x\right)_{\mathrm{n}}=n, \\
& g_{2} \cdot y_{\mathrm{s}}=\left(g_{2} \cdot y\right)_{\mathrm{s}}=c_{2}, \quad g_{2} \cdot y_{\mathrm{n}}=\left(g_{2} \cdot y\right)_{\mathrm{n}}=n,
\end{aligned}
$$

From (24) and Corollary 3.5 we obtain $\mathfrak{g}^{g_{1} \cdot x_{\mathrm{s}}}=\mathfrak{g}^{g_{2} \cdot y_{\mathrm{s}}}$ and $g_{1} \cdot x_{\mathrm{n}}=g_{2} \cdot y_{\mathrm{n}}$; whence (23) with $g=g_{2}^{-1} g_{1}$.

$(\mathrm{J}) \Rightarrow(\mathrm{P})$ Assume that $(23)$ holds for $x, y \in \mathfrak{g}_{1}$. By Proposition 3.8, there is a packet $G_{0} \cdot\left(\mathfrak{c}_{1}+n\right)$ in $\mathfrak{g}_{1}$ containing $y$; we have to show that $x \in G_{0} \cdot\left(\mathfrak{c}_{1}+n\right)$. By Definition 3.6, there are $c \in \mathfrak{c}_{1}$ and $h \in G_{0}$ such that $y=h \cdot(c+n)$; whence $y_{\mathrm{s}}=h \cdot c, y_{\mathrm{n}}=h \cdot n$. Plugging this in $(23)$, we obtain

$$
\mathfrak{g}^{w \cdot x_{\mathrm{s}}}=\mathfrak{g}^{c}, w \cdot x_{\mathrm{n}}=n, \quad \text { where } w=h^{-1} g .
$$

By Proposition 3.4( $\left.\mathrm{i}_{1}\right)$, we have $\mathfrak{t} \subset \mathfrak{g}^{c}$. Hence, by the first equality in (25), the semisimple element $w\left(x_{\mathrm{s}}\right)$ centralizes the maximal torus $\mathfrak{t}$. Whence $w \cdot x_{\mathrm{s}} \in \mathfrak{t}$ and, therefore, there is a unique cell $\mathfrak{c}^{\prime}$ in $\mathfrak{t}$ such that

$$
w \cdot x_{\mathrm{S}} \in \mathfrak{c}^{\prime} .
$$

From Proposition $3.4\left(\mathrm{i}_{2}\right)$ we then infer that $\mathfrak{z}\left(\mathfrak{g}^{w \cdot x_{\mathrm{s}}}\right)=\overline{\mathfrak{c}^{\prime}}$. On the other hand, by the same reason, $\mathfrak{z}\left(\mathfrak{g}^{c}\right)=\overline{\mathfrak{c}}$. Combining this with the first equality in (25), we conclude that $\overline{\mathfrak{c}^{\prime}}=\overline{\mathfrak{c}}$. By Proposition 3.2(iii), this yields

$$
\mathfrak{c}^{\prime}=\mathfrak{c} .
$$

From (26), (27), the second equality in (25), and Definition 3.6 we now conclude that $x \in G_{0} \cdot\left(\mathfrak{c}_{1}+n\right)$.

Corollary 3.11. The packets in $\mathfrak{g}_{1}$ do not depend on the choice of a torus $\mathfrak{t}$.

Corollary 3.12. Every two packets in $\mathfrak{g}_{1}$ are either equal or disjoint.

Proof. This is because property $(\mathrm{J})$ is clearly an equivalence relation on $\mathfrak{g}_{1}$ and, by Proposition 3.10 , packets in $\mathfrak{g}_{1}$ are precisely its equivalence classes.

Proposition 3.13. For every packet $G_{0} \cdot\left(\mathfrak{c}_{1}+n\right)$ and its closure $\overline{G_{0} \cdot\left(\mathfrak{c}_{1}+n\right)}$ in $\mathfrak{g}_{1}$, the following hold:

(i) $\mathfrak{g}_{i}^{x}=\mathfrak{g}_{i}^{\mathfrak{c}} \cap \mathfrak{g}_{i}^{n}$ for any $x \in \mathfrak{c}_{1}+n$ and $i$; 
(ii) $\left[\mathfrak{g}_{0}, x\right]=\left[\mathfrak{g}_{0}, y\right]$ for any $x, y \in \mathfrak{c}_{1}+n$.

(iii) $G_{0} \cdot\left(\mathfrak{c}_{1}+n\right)$ is irreducible and contains a dense open subset of $\overline{G_{0} \cdot\left(\mathfrak{c}_{1}+n\right)}$.

(iv) $G_{0} \cdot\left(\mathfrak{c}_{1}+n\right)$ is contained in a sheet of $\mathfrak{g}_{1}$.

(v) $\overline{G_{0} \cdot\left(\mathfrak{c}_{1}+n\right)}$ is an irreducible algebraic variety of dimension $d+\operatorname{dim} \mathfrak{c}_{1}$, where $d$ is the dimension of $G_{0}$-orbits in a sheet containing $G_{0} \cdot\left(\mathfrak{c}_{1}+n\right)$.

Proof. If $c \in \mathfrak{c}_{1}$ and $x=c+n$, then $c=x_{\mathrm{s}}$ and $n=x_{\mathrm{n}}$. This, the uniqueness of the Jordan decomposition, and Corollary 3.5 imply that

$$
\mathfrak{g}^{x}=\mathfrak{g}^{c} \cap \mathfrak{g}^{n}=\mathfrak{g}^{\mathfrak{c}} \cap \mathfrak{g}^{n} .
$$

Since $c, n, x$ are the homogeneous elements, $\mathfrak{g}^{c}, \mathfrak{g}^{n}, \mathfrak{g}^{x}$ are the graded subalgebras of $\mathfrak{g}$. Taking the $i$ th components, from (28) we obtain (i).

The subspace $\mathfrak{g}_{-1}$ is dual to $\mathfrak{g}_{1}$ with respect to $\langle\cdot, \cdot\rangle$, and the orthogonal complement in $\mathfrak{g}_{1}$ to $\mathfrak{g}_{-1}^{x}$ with respect to this duality is $\left[\mathfrak{g}_{0}, x\right]$; see [V76, Prop. 5]. Since $\mathfrak{g}_{-1}^{x}$, by (i), is the same for all $x \in \mathfrak{c}_{1}+n$, this proves (ii).

Corollary 3.3 and the connectedness of $G_{0}$ yield that $G_{0} \times \mathfrak{c}_{1}$ is an irreducible smooth affine algebraic variety. In view of Definition 3.6, the packet $G_{0} \cdot\left(\mathfrak{c}_{1}+n\right)$ is the image of the morphism:

$$
\varphi: G_{0} \times \mathfrak{c}_{1} \rightarrow \mathfrak{g}_{1}, \quad(g, c) \mapsto g \cdot(c+n) .
$$

By the general properties of morphisms, this implies (iii).

In view of (14), we have $\operatorname{dim} G_{0} \cdot x=\operatorname{dim} \mathfrak{g}_{0}-\operatorname{dim} \mathfrak{g}_{0}^{x}$. This and (i) show that $\operatorname{dim} G_{0} \cdot x$ is the same for all $x \in G_{0} \cdot\left(\mathfrak{c}_{1}+n\right)$, which, by (iii), means that $G_{0} \cdot\left(\mathfrak{c}_{1}+n\right) \subseteq{\overline{G_{0} \cdot\left(\mathfrak{c}_{1}+n\right)}}^{\mathrm{reg}}$. This implies (iv).

We may (and shall) consider $\varphi$ as a dominant morphism $G_{0} \times \mathfrak{c}_{1} \rightarrow \overline{G_{0} \cdot\left(\mathfrak{c}_{1}+n\right)}$. By [H77, Lem. 10.5], there is a point $z=(g, c) \in G_{0} \times \mathfrak{c}_{1}$ such that $\varphi(z)$ is a smooth point of $\overline{G_{0} \cdot\left(\mathfrak{c}_{1}+n\right)}$ and $d \varphi_{z}$ is a surjective map of the tangent spaces; this implies that $\operatorname{dim} \overline{G_{0} \cdot\left(\mathfrak{c}_{1}+n\right)}$ is equal to the dimension of the image of $d \varphi_{z}$. We shall now compute this latter dimension.

First note that since $\varphi$ is $G_{0}$-equivariant, we may (and shall) assume that $g$ is the identity element. From Proposition 3.2(ii) we infer that the closure $\overline{\mathfrak{c}_{1}}$ of $\mathfrak{c}_{1}$ in $\mathfrak{t}_{1}$ is a linear subspace, and $\overline{\mathfrak{c}_{1}} \backslash \mathfrak{c}_{1}$ is a union of finitely many hyperplanes. This shows that $\mathfrak{g}_{0} \oplus \overline{\mathfrak{c}_{1}}$ is the tangent space of $G_{0} \times \mathfrak{c}_{1}$ at $z$. Given this, we deduce from (14) and (29) that

$$
\text { the image of } d \varphi_{z} \text { is }\left[\mathfrak{g}_{0}, c+n\right]+\overline{\mathfrak{c}_{1}} \text {. }
$$

For the point $x:=c+n \in \mathfrak{c}_{1}+n$, we have $x_{\mathrm{s}}=c, x_{\mathrm{n}}=n$. Therefore,

$$
[\mathfrak{g}, x] \cap \mathfrak{z}\left(\mathfrak{g}^{c}\right)=0 ;
$$

see [TY05, Lem. 39.2.8]. But $\mathfrak{z}\left(\mathfrak{g}^{c}\right)=\overline{\mathfrak{c}}$ by Proposition 3.4(i $\left.\mathrm{i}_{2}\right)$. This and (31) yield $[\mathfrak{g}, x] \cap \overline{\mathfrak{c}}=0$; whence

$$
\left[\mathfrak{g}_{0}, x\right] \cap \overline{\mathfrak{c}_{1}}=0 .
$$

Combining (30), (32), (ii), and taking into account that $\left[\mathfrak{g}_{0}, x\right]$ is the tangent space to $G_{0} \cdot x$ at $x$, we now obtain that the dimension of the image of $d \varphi_{z}$ is equal to $\operatorname{dim}\left[\mathfrak{g}_{0}, x\right]+\operatorname{dim} \overline{\mathfrak{c}_{1}}=d+\operatorname{dim} \mathfrak{c}_{1}$. This completes the proof of $(\mathrm{v})$. 
Corollary 3.14. $\bmod \left(G_{0}: \overline{G_{0} \cdot\left(\mathfrak{c}_{1}+n\right)}\right)=\operatorname{dim} \mathfrak{c}_{1}$.

Lemma 3.15. $x \in\left(\mathfrak{z}\left(\mathfrak{g}^{x}\right)_{1}\right)^{\text {reg }}$ for every $x \in \mathfrak{g}_{1}$.

Proof. If $y \in \mathfrak{z}\left(\mathfrak{g}^{x}\right)$, then $\mathfrak{g}^{x} \subseteq \mathfrak{g}^{y}$; see [TY05, 35.3.2]. As $x$ is homogeneous, $\mathfrak{g}^{x}$ is graded. If $y$ is homogeneous, $\mathfrak{g}^{y}$ is graded as well, hence the specified inclusion yields $\mathfrak{g}_{i}^{x} \subseteq \mathfrak{g}_{i}^{y}$ for every $i$. In particular, $\mathfrak{g}_{0}^{x} \subseteq \mathfrak{g}_{0}^{y}$ for every $y \in \mathfrak{z}\left(\mathfrak{g}^{x}\right)_{1}$, whence $\operatorname{dim} G_{0} \cdot y \leqslant \operatorname{dim} G_{0} \cdot x$ and, by (4), the claim.

Lemma 3.16. Let $x \in \mathfrak{g}_{1}$ be a nilpotent element. Then

$$
G_{0} \cdot x \cap \mathfrak{z}\left(\mathfrak{g}^{x}\right)_{1}=\left(\mathfrak{z}\left(\mathfrak{g}^{x}\right)_{1}\right)^{\mathrm{reg}} .
$$

Proof. By by (4) and Lemma 3.15, for every $z \in \mathfrak{z}\left(\mathfrak{g}^{x}\right)_{1}$, we have

$$
z \in\left(\mathfrak{z}\left(\mathfrak{g}^{x}\right)_{1}\right)^{\mathrm{reg}} \Longleftrightarrow \operatorname{dim} G_{0} \cdot z=\operatorname{dim} G_{0} \cdot x .
$$

As $x$ is nilpotent and $\mathfrak{g}$ is semisimple, all elements of $\mathfrak{z}\left(\mathfrak{g}^{x}\right)$ are nilpotent (see [TY05, 35.1.2]). Therefore $\mathfrak{z}\left(\mathfrak{g}^{x}\right)_{1}$ lies in the variety of nilpotent elements of $\mathfrak{g}_{1}$. By [V76, Prop. 2], this variety is the union of finitely many $G_{0}$-orbits. From this we infer that among them there is an orbit $\mathcal{O}$ such that $\mathcal{O} \cap \mathfrak{z}\left(\mathfrak{g}^{x}\right)_{1}$ is a dense open subset of $\mathfrak{z}\left(\mathfrak{g}^{x}\right)_{1}$. This and (33) entail that

$$
\begin{aligned}
\overline{\mathcal{O} \cap \mathfrak{z}\left(\mathfrak{g}^{x}\right)_{1}} & =\mathfrak{z}\left(\mathfrak{g}^{x}\right)_{1}, \\
\mathcal{O} \cap \mathfrak{z}\left(\mathfrak{g}^{x}\right)_{1} & \subseteq\left(\mathfrak{z}\left(\mathfrak{g}^{x}\right)_{1}\right)^{\mathrm{reg}}, \\
\operatorname{dim} \mathcal{O} & =\operatorname{dim} G_{0} \cdot x .
\end{aligned}
$$

If $z \in\left(\mathfrak{z}\left(\mathfrak{g}^{x}\right)_{1}\right)^{\mathrm{reg}}$, then (33), (36) yield $\operatorname{dim} G_{0} \cdot z=\operatorname{dim} \mathcal{O}$. Since, by (34), $z \in \overline{\mathcal{O}}$, the latter equality implies that $G_{0} \cdot z=\mathcal{O}$. This equality and (35) yield

$$
\mathcal{O} \cap \mathfrak{z}\left(\mathfrak{g}^{x}\right)_{1}=\left(\mathfrak{z}\left(\mathfrak{g}^{x}\right)_{1}\right)^{\text {reg }} .
$$

From (37) and Lemma 3.15 we now infer that $\mathcal{O}=G_{0} \cdot x$. This equality and (37) complete the proof.

Proposition 3.17. For all $x, y \in \mathfrak{g}_{1}$, the following conditions are equivalent:

(i) $x$ and $y$ lie in the same packet in $\mathfrak{g}_{1}$.

(ii) There exists $g \in G_{0}$ such that $\mathfrak{g}^{y}=g \cdot \mathfrak{g}^{x}$.

(iii) There exists $g \in G_{0}$ such that $\mathfrak{z}\left(\mathfrak{g}^{y}\right)=g \cdot \mathfrak{z}\left(\mathfrak{g}^{x}\right)$.

Proof. The implication (ii) $\Rightarrow$ (iii) is clear.

Assume that (iii) holds. Then $\mathfrak{z}\left(\mathfrak{g}^{y}\right)=\mathfrak{z}\left(\mathfrak{g}^{g \cdot x}\right)$, which implies that $\mathfrak{g}^{y}=\mathfrak{g}^{g \cdot x}=$ $g \cdot \mathfrak{g}^{x}$; see [TY05, 35.3.2]. This proves the implication (iii) $\Rightarrow$ (ii).

Assume that (i) holds. Then by Proposition 3.10 there exists $g \in G_{0}$ such that (23) holds. Therefore

$$
\mathfrak{g}^{y}=\mathfrak{g}^{y_{\mathrm{s}}} \cap \mathfrak{g}^{y_{\mathrm{s}}}=g \cdot \mathfrak{g}^{x_{\mathrm{s}}} \cap g \cdot \mathfrak{g}^{x_{\mathrm{n}}}=g \cdot\left(\mathfrak{g}^{x_{\mathrm{s}}} \cap \mathfrak{g}^{x_{\mathrm{n}}}\right)=g \cdot \mathfrak{g}^{x} .
$$

This proves the implication (i) $\Rightarrow($ ii). 
Finally, let us prove that $x$ and $y$ lie same packet in $\mathfrak{g}_{1}$ assuming that (iii) holds. Since packets are $G_{0}$-stable, we may (and shall) assume that $g$ is the identity element, i.e.,

$$
\mathfrak{z}\left(\mathfrak{g}^{x}\right)=\mathfrak{z}\left(\mathfrak{g}^{y}\right) .
$$

According to [TY05, 39.1.1],

$$
\begin{aligned}
& \mathfrak{z}\left(\mathfrak{g}^{x}\right)=\mathfrak{z}\left(\mathfrak{g}^{x_{\mathrm{s}}}\right) \oplus \mathfrak{z}\left(\left[\mathfrak{g}^{x_{\mathrm{s}}}, \mathfrak{g}^{x_{\mathrm{s}}}\right]^{x_{\mathrm{n}}}\right), \\
& \mathfrak{z}\left(\mathfrak{g}^{y}\right)=\mathfrak{z}\left(\mathfrak{g}^{y_{\mathrm{s}}}\right) \oplus \mathfrak{z}\left(\left[\mathfrak{g}^{y_{\mathrm{s}}}, \mathfrak{g}^{y_{\mathrm{s}}}\right]^{y_{\mathrm{n}}}\right),
\end{aligned}
$$

and the first (respectively, second) direct summand in each of the equalities (39) is the set of all semisimple (respectively, nilpotent) elements of the lefthand side of this equality. This and (38) then entail

$$
\begin{aligned}
\mathfrak{z}\left(\mathfrak{g}^{x_{\mathrm{s}}}\right) & =\mathfrak{z}\left(\mathfrak{g}^{y_{\mathrm{s}}}\right), \\
\mathfrak{z}\left(\left[\mathfrak{g}^{x_{\mathrm{s}}}, \mathfrak{g}^{x_{\mathrm{s}}}\right]^{x_{\mathrm{n}}}\right) & =\mathfrak{z}\left(\left[\mathfrak{g}^{y_{\mathrm{s}}}, \mathfrak{g}^{y_{\mathrm{s}}}\right]^{y_{\mathrm{n}}}\right) .
\end{aligned}
$$

Since $x$ and $y$ are homogeneous elements, the left- and right-hand sides of (40) are the graded subalgebras of $\mathfrak{g}$. The subalgebras $\left[\mathfrak{g}^{x_{\mathrm{s}}}, \mathfrak{g}^{x_{\mathrm{s}}}\right],\left[\mathfrak{g}^{y_{\mathrm{s}}}, \mathfrak{g}^{y_{\mathrm{s}}}\right]$ are semisimple and $x_{\mathrm{n}} \in\left[\mathfrak{g}^{x_{\mathrm{s}}}, \mathfrak{g}^{x_{\mathrm{s}}}\right]_{1}, y_{\mathrm{n}} \in\left[\mathfrak{g}^{y_{\mathrm{s}}}, \mathfrak{g}^{y_{\mathrm{s}}}\right]_{1}$.

Let $H$ be the connected closed subgroup of $G_{0}$ such that Lie $H=\left[\mathfrak{g}^{x_{\mathrm{s}}}, \mathfrak{g}^{x_{\mathrm{s}}}\right]_{0}$. Applying Lemma 3.16, in which $x$ and $\mathfrak{g}$ are replaced respectively by $x_{\mathrm{n}}$ and $\left[\mathfrak{g}^{x_{\mathrm{s}}}, \mathfrak{g}^{x_{\mathrm{s}}}\right]$, and taking (40) into account, we obtain:

$$
H \cdot x_{\mathrm{n}} \cap \mathfrak{z}\left(\left[\mathfrak{g}^{x_{\mathrm{s}}}, \mathfrak{g}^{x_{\mathrm{s}}}\right]^{x_{\mathrm{n}}}\right)_{1}=\left(\mathfrak{z}\left(\left[\mathfrak{g}^{x_{\mathrm{s}}}, \mathfrak{g}^{x_{\mathrm{s}}}\right]^{x_{\mathrm{n}}}\right)_{1}\right)^{\mathrm{reg}} \stackrel{(40)}{=}\left(\mathfrak{z}\left(\left[\mathfrak{g}^{y_{\mathrm{s}}}, \mathfrak{g}^{y_{\mathrm{s}}}\right]^{y_{\mathrm{n}}}\right)_{1}\right)^{\mathrm{reg}},
$$

where the notation $X^{\text {reg }}$ refers to the action of $H$ on $\left[\mathfrak{g}^{x_{\mathrm{s}}}, \mathfrak{g}^{x_{\mathrm{s}}}\right]_{1}$.

By Lemma 3.15, the element $y_{\mathrm{n}}$ lies in the right-hand side of (41). Therefore (41) implies that there exists $g \in H$ such that

$$
g \cdot x_{\mathrm{n}}=y_{\mathrm{n}} .
$$

Since $\left[\mathfrak{g}^{x_{\mathrm{s}}}, \mathfrak{g}^{x_{\mathrm{s}}}\right]$ and $\mathfrak{z}\left(\mathfrak{g}^{x_{\mathrm{s}}}\right)$ commute, $H$ acts trivially on $\mathfrak{z}\left(\mathfrak{g}^{x_{\mathrm{s}}}\right)$. This and the first equality in (40) yield

$$
\mathfrak{z}\left(\mathfrak{g}^{g \cdot x_{\mathrm{s}}}\right)=g \cdot \mathfrak{z}\left(\mathfrak{g}^{x_{\mathrm{s}}}\right)=\mathfrak{z}\left(\mathfrak{g}^{x_{\mathrm{s}}}\right) \stackrel{(40)}{=} \mathfrak{z}\left(\mathfrak{g}^{y_{\mathrm{s}}}\right) .
$$

From (43), using [TY05, 35.3.2], we infer that

$$
\mathfrak{g}^{g \cdot x_{\mathrm{s}}}=\mathfrak{g}^{y_{\mathrm{s}}} .
$$

By Proposition 3.10, the equalities (42) and (44) imply (i), thereby completing the proof of implication (iii) $\Rightarrow(\mathrm{i})$.

Using Proposition 3.17, we now can show that in fact a property stronger than Proposition 3.13(iii) holds:

Proposition 3.18. Every packet $P$ in $\mathfrak{g}_{1}$ is open in its closure in $\mathfrak{g}_{1}$. 
Proof. By Proposition 3.13, there exists an integer $d$ such that $P$ lies in the locally closed subset $X=\left\{x \in \mathfrak{g} \mid \operatorname{dim} \mathfrak{g}^{x}=d\right\}$ of $\mathfrak{g}$. Let $\operatorname{Grass}(\mathfrak{g}, d)$ be the Grassmannian of $d$-dimensional linear subspaces in $\mathfrak{g}$ endowed with the natural action of $G$. The map

$$
\varphi: X \rightarrow \operatorname{Grass}(\mathfrak{g}, d), \quad x \mapsto \mathfrak{g}^{x} .
$$

is $G$-equivariant. Let $x \in P$. Then Proposition 3.17 implies that

$$
P=\mathfrak{g}_{1} \cap \varphi^{-1}\left(G_{0} \cdot \varphi(x)\right) .
$$

Since $\varphi$ is a morphism (see [TY05, 19.7.6 and 29.3.1]) and orbits of algebraic transformation groups are open in their closures, (45) shows that $P$ enjoys the latter property as well.

3.6. We conclude with describing the relationship between the sheets and the packets in $\mathfrak{g}_{1}$. Below bar stands for the closure in $\mathfrak{g}_{1}$.

Proposition 3.19. For every sheet $S$ of $\mathfrak{g}_{1}$, there is a unique packet $P$ in $\mathfrak{g}_{1}$ such that $P \subseteq S$ and $\bar{P}=\bar{S}$. Moreover, $S=\bar{P}^{\text {reg }}$.

Proof. If $d$ is the dimension of $G_{0}$-orbits in $S$, then by Propositions $3.8,3.13$ and in view of the definition of a sheet, we have

$$
\left\{x \in \mathfrak{g}_{1} \mid \operatorname{dim} G_{0}(x)=d\right\}=\bigcup_{i=1}^{p} S_{i}=\bigcup_{j=i}^{q} P_{j},
$$

where $S_{1}, \ldots, S_{p}$ and $P_{1}, \ldots, P_{q}$ are, respectively, some pairwise different sheets and packets in $\mathfrak{g}_{1}$; the sheet $S$ is one of $S_{1}, \ldots, S_{m}$. Whence

$$
\bigcup_{i=1}^{p} \overline{S_{i}}=\bigcup_{j=i}^{q} \overline{P_{j}} \text {. }
$$

As sheets and packets contain open subsets of their closures, we have $\overline{S_{i}} \nsubseteq \overline{S_{j}}$ and $\overline{P_{i}} \nsubseteq \overline{P_{j}}$ for all $i \neq j$. Since all $\overline{S_{i}}$ and $\overline{P_{j}}$ are irreducible, (46) then implies that $p=q$ and there is a permutation $\sigma$ of $\{1, \ldots, p\}$ such that $\overline{S_{i}}=\overline{P_{\sigma(i)}}$.

The last claim of this proposition follows from the definition of a regular sheet.

\section{REFERENCES}

[A75] V. I. Arnold, Critical points of smooth functions, in: Proceedings of the International Congress of Mathematicians, Vol. 1 (Vancouver, BC, 1974), Canad. Math. Congress, Montreal, Que., 1975, pp. 19-39.

[B68] N. Bourbaki, Groupes et Algèbres de Lie, Chap. IV, V, VI, Hermann, Paris, 1968.

[B91] A. Borel, Linear Algebraic Groups, 2nd enlarged ed., Graduate Texts in Mathematics, Vol. 126, Springer-Verlag, 1991.

[H77] R. Hartshorne, Algebraic Geometry, Springer-Verlag, New York, 1977.

[J62] N. Jacobson, Lie Algebras, Interscience Tracts in Pure and Applied Mathematics, No. 10, Interscience Publishers, New York, 1962.

[K75] V. G. Kac, On the question of describing the orbit space of linear algebraic groups, Uspekhi Mat. Nauk 30 (1975), no. 6(186), 173-174 (in Russian).

[K801] V. G. Kac, Some remarks on nilpotent orbits, J. Algebra 64 (1980), 190-213.

$\left[\mathrm{K}_{2}\right] \quad$ G. Kempf, Some quotient surfaces are smooth, Michigan Math. J. 27 (1980), 295299. 
[L89] P. Littelmann, Koreguläre und äquidimensionale Darstellungen, J. Algebra 123 (1989), 193-222.

[KPV76] V. G. Kac, V. L. Popov, É. B. Vinberg, Sur les groupes linéaires algébriques dont l'algèbre des invariants est libre, C. R. Acad. Sci. Paris Sér. A 283 (1976), A875A878.

[KKY86] T. Kimura, S.-I. Kasai, O. Yasukura, A classification of the representations of reductive algebraic groups which admit only a finite number of orbits, Amer. J. Math. 108 (1986), no. 3, 643-691.

[P71] V. L. Popov, A stability criterion for an action of a semisimple group of a factorial variety, Math USSR, Izv. 4 (1971), 527-535.

[P74] V. L. Popov, Structure of the closure of orbits in spaces of finite-dimensional linear SL(2) representations, Math. Notes of the Academy of Sciences of the USSR 16 (1974), no. 6, 1159-1162.

[P77] V. L. Popov, Representations with a free module of covariants, Funct. Anal. Appl. 10 (1977), 242-244.

[P80] V. L. Popov, Classification of spinirs of dimension fourteen, Trans. Moscow Math. Soc. (1980), no. 1, 181-232.

[P08] V. L. Popov, Irregular and singular loci of commuting varieties, Transformation Groups 13 (2008), no. 3-4, special issue dedicated to Bertram Kostant on the occasion of his 80th birthday (2008), 819-837.

[P171] В. Л. Попов, О модальности представлений, Доклады Академии Наук 475 (2017), no. 1, 14-16.

[P172] V. L. Popov, Algebraic groups whose orbit closures contain only finitely many orbits, arXiv:1707.06914v1 [math.AG] 21 Jul 2017.

[PV94] V. L. Popov, E. B. Vinberg, Invariant Theory, in: Algebraic Geometry IV, Enc. Math. Sci., Vol. 55, Springer, Berlin, 1994, pp. 123-284.

[SK77] M. Sato, T. Kimura, A classification of irreducible prehomogeneous vector spaces and their relative invariants, Nagoya Math. J. 65 (1977), 1-155.

[TY05] P. Tauvel, R. W. T. Yu, Lie Algebras and Algebraic Groups, Springer Manographs in Mathematics, Springer, Berlin, 2005.

[V76] É. B. Vinberg, The Weyl group of a graded Lie algebra, Math. USSR-Izv. 10 (1976), no. 3, 463-495.

[V86] É. B. Vinberg, Complexity of action of reductive groups, Funct. Analysis Appl. 20 1986, no. 1, 1-11.

Steklov Mathematical Institute, Russian Academy of Sciences, Gubkina 8, Moscow 119991, Russia

National Research University, Higher School of Economics, Myasnitskaya 20, Moscow 101000, Russia

E-mail address: popovvl@mi.ras.ru 